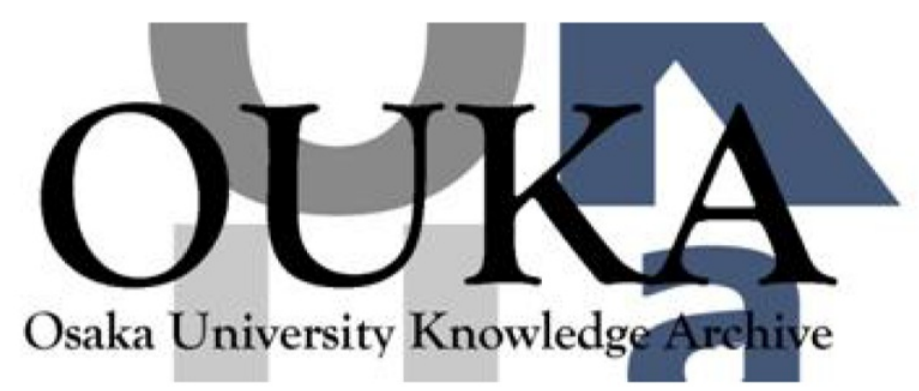

\begin{tabular}{|c|l|}
\hline Title & $\begin{array}{l}\text { Regional heterogeneity of afterload sensitivity } \\
\text { in myocardial strain }\end{array}$ \\
\hline Author(s) & $\begin{array}{l}\text { Asanuma, Toshihiko; Nakayama, Tomohito; Masuda, } \\
\text { Kasumi et al. }\end{array}$ \\
\hline Citation & $\begin{array}{l}\text { Journal of Medical Ultrasonics. 47(4) p. 501- } \\
\text { p. } 506\end{array}$ \\
\hline Issue Date & $2020-08-08$ \\
\hline oaire:version AM \\
\hline URL & https://hdl. handle. net/11094/86332 \\
\hline rights & \\
\hline Note & \\
\hline
\end{tabular}

Osaka University Knowledge Archive : OUKA

https://ir. Library. osaka-u. ac. jp/

Osaka University 
[Original article]

\section{Regional heterogeneity of afterload sensitivity in myocardial strain}

Toshihiko Asanuma, Tomohito Nakayama, Kasumi Masuda, Serina Takeda, Satoshi Nakatani

Division of Functional Diagnostics, Department of Health Sciences, Osaka University

Graduate School of Medicine, 1-7 Yamadaoka, Suita, Osaka 565-0871, Japan.

Corresponding author:

Toshihiko Asanuma

Division of Functional Diagnostics, Department of Health Sciences, Osaka University

Graduate School of Medicine, 1-7 Yamadaoka, Suita, Osaka, 565-0871, Japan

E-mail: toshi@sahs.med.osaka-u.ac.jp 


\begin{abstract}
Purpose The peak systolic strain decreases due to afterload augmentation. However, its deterioration (i.e., afterload sensitivity) may be different within left ventricular (LV) segments. We investigated how afterload influences regional strain and whether there is regional heterogeneity of afterload sensitivity.
\end{abstract}

Methods Afterload was increased by aortic banding in 20 open-chest dogs. Short-axis images were acquired at baseline and during banding. Circumferential strain was analyzed in six segments, and the absolute decrease in the peak systolic strain during banding $(\Delta \varepsilon)$ was calculated for each segment. To assess the effect of the compensatory preload recruitment during banding, the endocardial lengths of the septum and free wall were measured at enddiastole, and the rate of increase due to banding was calculated.

Results LV systolic pressure was significantly increased during banding ( $100 \pm 14$ vs. $143 \pm 18$ $\mathrm{mmHg}, P<0.001)$. The peak systolic strain in all segments was significantly decreased during banding. $\Delta \varepsilon$ in the anterior segment, which is a part of the free wall, was significantly lower than that in the inferoseptal segment $(2.6 \pm 4.7$ vs. $6.5 \pm 3.5 \%, P=0.035)$. The rate of increase in endocardial length in the free wall was significantly larger than that in the septum $(15.6 \pm 10.4$ vs. $8.1 \pm 7.4 \%, P=0.014)$.

Conclusion The decrease in septal strain during afterload augmentation was larger than that in free wall strain, indicating that there was regional heterogeneity of afterload sensitivity in circumferential strain. The larger compensatory preload recruitment in the free wall than in the septum is implicated as a cause of the heterogeneity.

\title{
Keywords
}

afterload, echocardiography, myocardial strain, post-systolic shortening, speckle tracking 


\section{Introduction}

Quantitative assessment of left ventricular (LV) contraction is essential when evaluating LV systolic function; however, LV contraction is also affected by loading conditions in addition to its own intrinsic contractility $[1,2]$. Afterload modification, in particular, should be carefully examined when assessing patients with hypertension or aortic stenosis. LV stroke volume and ejection fraction (EF), which are important indices for global systolic function, are easily decreased during afterload augmentation $[3,4]$.

Afterload modification can also be observed for regional function. Myocardial strain analysis using speckle-tracking echocardiography allows the quantitative assessment of regional LV function [5-7]. The peak systolic strain, which is used as an index of regional systolic function, is decreased by increased afterload as well as EF [7-9]. However, its deterioration during afterload augmentation (i.e., afterload sensitivity) may be different among LV segments due to the complex morphology of the LV. We therefore investigated how afterload influences regional strain and whether there is regional heterogeneity of afterload sensitivity. Furthermore, we explored the possibility of a mechanism controlling the heterogeneity.

\section{Materials and Methods}

\section{Animal preparation}

All animal studies were approved by the animal experimentation committee and performed in accordance with the guidelines for the care and use of laboratory animals at our institution. A total of 20 open-chest dogs $(10.6 \pm 1.6 \mathrm{~kg})$ were used in this study. The dogs were anesthetized using intramuscular injection of xylazine $(0.5 \mathrm{mg} / \mathrm{kg})$ followed by intravenous administration of pentobarbital $(25.9 \mathrm{mg} / \mathrm{kg})$, and were then intubated and ventilated using a respirator. Oxygen saturation was monitored with a pulse oximeter and maintained within normal ranges. Anesthesia was maintained through continuous infusion of pentobarbital (6 $\mathrm{mg} / \mathrm{kg} / \mathrm{h})$ and midazolam $(0.18 \mathrm{mg} / \mathrm{kg} / \mathrm{h})$ throughout the experiment. Buprenorphine (4 $\mu \mathrm{g} / \mathrm{kg}$ ) was administered intramuscularly as an analgesic. The electrocardiogram was continuously monitored, and LV pressure was measured using a 5-Fr micromanometer 
(Millar Instruments, Houston, TX, USA) inserted through the right femoral artery or the right carotid artery into the LV. The heart was suspended in a pericardial cradle through a left parasternal thoracotomy.

\section{Echocardiography}

Echocardiography was performed using an Aplio Artida ultrasound system with a PST-50BT or 30BT transducer (Canon Medical Systems, Otawara, Japan). The transmitting and receiving frequencies were set at 2.9 and 5.8 $\mathrm{MHz}(50 \mathrm{BT})$ and 1.8 and $3.6 \mathrm{MHz}$ (30BT), respectively, and the frame rate was set at 77-99 frames/s. LV short-axis images at the papillary muscle were visualized with a water bath as a standoff. A microphone for the phonocardiogram was placed on the base of the aorta to determine the timing of aortic valve closure (AVC) [10].

\section{Experimental protocol}

Hemodynamic and echocardiographic data were simultaneously acquired at baseline. Acute afterload augmentation was then performed by banding the upper descending aorta (aortic banding), producing a 30- to 70-mmHg increase in LV systolic pressure. Both measurements were again acquired after $5 \mathrm{~min}$ of stabilization during aortic banding.

\section{Data analysis}

Heart rate, LV systolic pressure, LV end-diastolic pressure, maximum and minimum time derivatives of LV pressure ( $\left.\mathrm{dP} / \mathrm{dt}_{\max }, \mathrm{dP} / \mathrm{dt}_{\min }\right)$, and the time constant of $\mathrm{LV}$ pressure decay during the isovolumic relaxation period $(\tau)$ were averaged from five consecutive cardiac cycles. LV end-diastolic diameter, LV end-systolic diameter, and \% fractional shortening were averaged from two or three consecutive cardiac cycles. Midwall systolic stress in the circumferential direction was calculated from the formula previously described by Murai, et al. [9].

Two-dimensional speckle-tracking analysis was performed using the offline software Ultra Extend (Canon Medical Systems, Otawara, Japan). End-diastole and end-systole were 
defined at the beginning of the QRS complex of the electrocardiogram and the timing of AVC, respectively. The endocardial border excluding papillary muscles was manually traced at end-systole, and the epicardial border was determined by setting an even width of myocardium. Endocardial and epicardial borders were automatically tracked for all frames during a cardiac cycle. Global circumferential strain (GCS) and regional circumferential strain in six segments were analyzed as the length change on the endocardial border. If required, each segment was manually adjusted according to the segment definitions published by the EACVI/ASE/Industry Task Force (Fig. 1) [11]. The peak systolic strain was measured from two or three consecutive cardiac cycles and averaged, and the absolute decrease in the peak systolic strain during aortic banding $(\Delta \varepsilon)$ was calculated. The reproducibility of regional radial strain is comparably inferior to that of regional circumferential strain due to the difficulty in tracking the epicardium [12]. During this study, radial strain was not analyzed because high reproducibility was required for the evaluation of $\Delta \varepsilon$.

Changes in regional wall stress can affect regional afterload sensitivity. Although it is difficult to exactly estimate the change in regional wall stress in the LV, the radius of curvature can be assessed. Therefore, the radius of curvature at end-systole was measured separately in the septum and free wall, and the rate of increase during aortic banding was calculated in 10 dogs, in which the endocardium of interest could be clearly detected and approximated as an arc. When afterload is acutely augmented in the LV, preload also increases to compensate for the decrease in stroke volume [2]. To assess the effect of this compensatory preload recruitment, the endocardial lengths at end-diastole were measured separately in the septum and free wall, and the rate of increase during aortic banding was calculated in the 10 dogs mentioned above.

\section{Statistical analysis}

Data are expressed as mean \pm standard deviation. Comparison of baseline values and those during aortic banding was performed using paired $t$ test. Multiple comparisons of $\Delta \varepsilon$ were performed using one-way analysis of variance with Tukey HSD test (in homogeneity of 
variance) or Games-Howell test (in heterogeneity of variance). Values of $P<0.05$ were considered to represent statistical significance.

\section{Results}

\section{Hemodynamics and echocardiographic data}

During aortic banding, LV systolic pressure and LV end-diastolic pressure were significantly increased, and heart rate and $\mathrm{dP} / \mathrm{dt}_{\min }$ were significantly decreased, and $\tau$ was significantly prolonged. In terms of echocardiographic data, LV end-diastolic and end-systolic diameters were significantly increased, and \% fractional shortening was significantly decreased. Midwall systolic stress was significantly increased (Table 1).

\section{Circumferential strain}

Representative circumferential strain curves at baseline and during aortic banding are shown in Fig. 2. In the inferoseptal segment, the peak systolic strain was markedly decreased during aortic banding compared to baseline, and post-systolic shortening (PSS), which is myocardial shortening after AVC, was observed. In the anterior and anterolateral segments, however, the decreases in the peak systolic strain appeared to be small.

Across all data, the absolute peak systolic strain in each segment was significantly decreased during aortic banding compared to baseline (Table 2). $\Delta \varepsilon$ in the anterior segment, which is a part of the free wall, was significantly lower, and that in the anterolateral segment tended to be lower than that in the inferoseptal segment (Fig. 3). In terms of the rate of increase in the radius of curvature during aortic banding, there was no significant difference between the septum and free wall $(28.3 \pm 19.5$ vs. $25.4 \pm 16.1 \%, P=0.639)$.

\section{Compensatory preload recruitment}

The LV was rapidly dilated during aortic banding due to a compensatory reaction (Fig. 4). The rate of increase in the endocardial length in the free wall was significantly larger than that in the septum (Figs. 4 and 5). 


\section{Discussion}

In this study, we investigated a possible regional heterogeneity of afterload sensitivity in circumferential strain. The results indicated that, although regional strain was decreased in all segments during aortic banding, $\Delta \varepsilon$ in the anterior and anterolateral segments was lower than that in the inferoseptal segment. The compensatory increase in endocardial length during aortic banding was larger in the free wall than in the septum.

It is well known that afterload augmentation decreases regional strain [7, 8]; however, whether there is regional heterogeneity of afterload sensitivity in strain has not been fully evaluated. Recently, Murai et al. examined the impact of afterload augmentation induced by handgrip stress on global and regional strain in healthy subjects and excellently demonstrated that regional strain linearly and inversely correlated with wall stress [9]. However, comparisons of afterload sensitivity between segments were not performed in their study.

The results in our study indicate that there is regional heterogeneity of afterload sensitivity in circumferential strain. As shown in Fig. 2, PSS in the inferoseptal segment during aortic banding suggests greater deterioration of myocardial contraction in this segment than other segments because PSS occurs in the myocardium with contractile dysfunction through an imbalance of tension between the myocardium [13].

Although regional differences in wall stress are thought to be an essential cause of varied afterload sensitivity, it is difficult to estimate regional wall stress due to convoluted myocardial fiber orientation, uneven LV geometry, and the presence of the right ventricle. However, if compensatory preload recruitment during acute afterload augmentation is different among the segments, it may be a reason for regional heterogeneity. Hence, we evaluated the endocardial length in the septum and free wall before and during afterload augmentation and found that the length increase was larger in the free wall than in the septum. This result suggests that the difference in preload recruitment may be one of the reasons why the decreases in anterior and anterolateral strain, which are part of the free wall, were lower. The septum was unlikely to generate preload recruitment due to the presence of the right ventricle.

Conversely, Stewart et al. investigated the changes in strain during bicycle exercise and 
showed that the increase in lateral strain was lower than that in septal strain [14]. Although bicycle exercise is different from our model because cardiac output increases and systemic vascular resistance decreases, their results also suggest that there is regional heterogeneity in strain during stress.

Whether there is regional heterogeneity in the chronic increase in afterload was not evaluated in this study. It is still unclear for circumferential strain, but regional heterogeneity may be small in the chronic increase because compensatory preload recruitment does not occur and wall stress can be compensated by LV hypertrophy. Meanwhile, in the case of longitudinal strain, it has been reported that the decrease in the strain in the septal wall is greater than that in the lateral wall in patients with hypertension [15] and in a chronic aortic banding pig model [16]. This is thought to be due to higher wall stress in the septum, which has a larger radius of curvature in the longitudinal section [17].

\section{Clinical implications}

The present study was conducted to resolve a pending physiological issue in myocardial strain. When circumferential strain is analyzed in patients with acute hypertension, attention to regional heterogeneity of afterload sensitivity is required. As circumferential strain in the septum appears to be more sensitive to increased afterload than that in the free wall, strain analysis in the septal segment may be useful to detect mild afterload mismatch.

\section{Limitations}

First, we measured endocardial length in the septum and free wall to assess compensatory preload recruitment because junctions of the right ventricular wall could be used as landmarks. For more detailed analysis, however, change in endocardial length in each segment should have been measured by implanted markers. The relationship between the rate of increase in endocardial length and $\Delta \varepsilon$ in each segment should also be evaluated in a further study.

Second, in our dog model, longitudinal strain tends to represent lower values than those in humans at baseline conditions [18]. Moreover, it was hard to acquire the same views at 
baseline and during aortic banding because immobilization of the transducer was difficult in apical views. Therefore, we did not evaluate regional heterogeneity in longitudinal strain in this study.

Finally, the use of a pericardial cradle in the open-chest experiment allows acquisition of clear and stable echocardiographic images. Although we performed pericardiotomy for this preparation, it may have caused larger preload recruitment in the free wall. Further studies in closed-chest models are required.

\section{Conclusion}

There was regional heterogeneity of afterload sensitivity in circumferential strain. Larger compensatory preload recruitment in the free wall than in the septum is implicated as a cause of the heterogeneity. 


\section{Ethical statements}

All institutional and national guidelines for the care and use of laboratory animals were followed.

\section{Conflict of interest}

This research was supported by the research and development committee program of the Japan Society of Ultrasonics in Medicine.

Toshihiko Asanuma, Tomohito Nakayama, Kasumi Masuda, and Serina Takeda declare that they have no conflicts of interest. Satoshi Nakatani received a research grant from Canon Medical Systems. 


\section{References}

1. Cohn JN. Blood pressure and cardiac performance. Am J Med. 1973;55:351-61.

2. Ross J Jr. Cardiac function and myocardial contractility: a perspective. J Am Coll Cardiol. 1983;1:52-62.

3. MacGregor DC, Covell JW, Mahler F, et al. Relations between afterload, stroke volume, and descending limb of Starling's curve. Am J Physiol. 1974;227:884-90.

4. Gunther S, Grossman W. Determinants of ventricular function in pressure-overload hypertrophy in man. Circulation. 1979;59:679-88.

5. Amundsen BH, Helle-Valle T, Edvardsen T, et al. Noninvasive myocardial strain measurement by speckle tracking echocardiography: validation against sonomicrometry and tagged magnetic resonance imaging. J Am Coll Cardiol. 2006;47:789-93.

6. Klaeboe LG, Edvardsen T. Echocardiographic assessment of left ventricular systolic function. J Echocardiogr. 2019;17:10-6.

7. Voigt JU, Cvijic M. 2- and 3-dimensional myocardial strain in cardiac health and disease. JACC Cardiovasc Imaging. 2019;12:1849-63.

8. Donal $\mathrm{E}$, Bergerot $\mathrm{C}$, Thibault $\mathrm{H}$, et al. Influence of afterload on left ventricular radial and longitudinal systolic functions: a two-dimensional strain imaging study. Eur J Echocardiogr. 2009;10:914-21.

9. Murai D, Yamada S, Hayashi T, et al. Relationships of left ventricular strain and strain rate to wall stress and their afterload dependency. Heart Vessels. 2017;32:574-83.

10. Masuda K, Asanuma T, Taniguchi A, et al. Assessment of dyssynchronous wall motion during acute myocardial ischemia using velocity vector imaging. JACC Cardiovasc Imaging 2008; 1:210-20.

11. Voigt JU, Pedrizzetti G, Lysyansky P, et al. Definitions for a common standard for 2D speckle tracking echocardiography: consensus document of the EACVI/ASE/Industry Task Force to standardize deformation imaging. Eur Heart J Cardiovasc Imaging. 2015;16:1-11.

12. Sakurai D, Asanuma T, Masuda K, et al. Myocardial layer-specific analysis of ischemic memory using speckle tracking echocardiography. Int J Cardiovasc Imaging. 
2014;30:739-48.

13. Asanuma T, Nakatani S. Myocardial ischaemia and post-systolic shortening. Heart. 2015;101:509-16

14. Stewart GM, Chan J, Yamada A, et al. Impact of high-intensity endurance exercise on regional left and right ventricular myocardial mechanics. Eur Heart J Cardiovasc Img. 2017;18:688-96.

15. Baltabaeva A, Marciniak M, Bijnens B, et al. Regional left ventricular deformation and geometry analysis provides insights in myocardial remodeling in mild to moderate hypertension. 2008;9:501-8.

16. Reant P, Metras A, Detaille D, et al. Impact of afterload increase on ventricular myocardial deformation indices. J Am Soc Echocardiogr. 2016;29:1217-28.

17. Heng MK, Janz RF, Jobin J. Estimation of regional stress in the left ventricular septum and free wall: an echocardiographic study suggesting a mechanism for asymmetric septal hypertrophy. Am Heart J. 1985;110:84-90.

18. Hioki A, Asanuma T, Masuda K, et al. Detection of abnormal myocardial deformation during acute myocardial ischemia using three-dimensional speckle tracking echocardiography. J Echocardiogr. 2020;18:57-66. 


\section{Figure legends}

Fig. 1 Segmentation in the left ventricular (LV) short-axis view. Global circumferential strain and regional strain in six segments (anteroseptal, inferoseptal, inferior, inferolateral, anterolateral, and anterior) were analyzed as the length change on the endocardial border. Each segment was manually adjusted according to segment definitions published by the EACVI/ASE/Industry Task Force [11].

Fig. 2 Representative global circumferential strain (GCS) and regional strain curves at baseline (a) and during aortic banding (b). In the inferoseptal segment (purple), the peak systolic strain was decreased during aortic banding compared to baseline. The decrease in systolic strain was followed by post-systolic shortening, which is myocardial shortening after aortic valve closure (AVC).

Fig. 3 The absolute decrease in the peak systolic strain during aortic banding $(\Delta \varepsilon)$ in each segment. There was regional heterogeneity in $\Delta \varepsilon$. $\Delta \varepsilon$ in the anterior segment, which is a part of the free wall, was significantly lower, and that in the anterolateral segment tended to be lower than that in the inferoseptal segment. $* P<0.05$ vs. inferoseptal.

Fig. 4 End-diastolic short-axis images at baseline (a) and during aortic banding (b). The left ventricle was rapidly dilated during aortic banding because of compensatory preload recruitment. The endocardial length in the free wall (light blue) was increased more than that in the septum (purple).

Fig. 5 The rate of increase in the endocardial length in the septum and the free wall. The rate of increase in the free wall was significantly larger than that in the septum. $* P<0.05$ vs. septum. 
Table 1 Hemodynamics and echocardiographic data

\begin{tabular}{lccc}
\hline & Baseline & Aortic banding & $P$ value \\
\hline Heart rate (bpm) & $137 \pm 17$ & $121 \pm 14$ & $<0.001$ \\
LV systolic pressure (mmHg) & $100 \pm 14$ & $143 \pm 18$ & $<0.001$ \\
LV end-diastolic pressure (mmHg) & $5 \pm 2$ & $8 \pm 3$ & $<0.001$ \\
$\mathrm{dP} / \mathrm{dt}_{\max }(\mathrm{mmHg} / \mathrm{s})$ & $2261 \pm 510$ & $2301 \pm 567$ & 0.671 \\
$\mathrm{dP} / \mathrm{dt}_{\min }(\mathrm{mmHg} / \mathrm{s})$ & $-2000 \pm 587$ & $-2545 \pm 574$ & $<0.001$ \\
$\tau(\mathrm{ms})$ & $37 \pm 8$ & $52 \pm 14$ & $<0.001$ \\
$\mathrm{LV}$ end-diastolic diameter $(\mathrm{mm})$ & $27 \pm 2$ & $33 \pm 4$ & $<0.001$ \\
$\mathrm{LV}$ end-systolic diameter $(\mathrm{mm})$ & $18 \pm 2$ & $26 \pm 5$ & $<0.001$ \\
$\%$ fractional shortening $(\%)$ & $33 \pm 3$ & $20 \pm 6$ & $<0.001$ \\
Midwall systolic stress $\left(\mathrm{dyn} / \mathrm{mm}^{2}\right)$ & $511 \pm 156$ & $1621 \pm 641$ & $<0.001$ \\
\hline
\end{tabular}


Table 2 Peak systolic strain (absolute value)

\begin{tabular}{lccc}
\hline & Baseline & Aortic banding & $P$ value \\
\hline Global circumferential strain (\%) & $17 \pm 3$ & $13 \pm 3$ & $<0.001$ \\
Inferior (\%) & $16 \pm 3$ & $10 \pm 3$ & $<0.001$ \\
Inferolateral (\%) & $15 \pm 4$ & $11 \pm 3$ & 0.001 \\
Anterolateral (\%) & $16 \pm 4$ & $13 \pm 4$ & 0.010 \\
Anterior (\%) & $18 \pm 4$ & $16 \pm 4$ & 0.024 \\
Anteroseptal (\%) & $20 \pm 3$ & $16 \pm 4$ & $<0.001$ \\
Inferoseptal (\%) & $18 \pm 2$ & $12 \pm 3$ & $<0.001$ \\
\hline
\end{tabular}

